Commentary on Borsboom et al., BBS Vol. 41, 2018

\title{
The adaptive self: Culture and social flexibility in feedback networks
}

Daina Crafa ${ }^{a}$ and Saskia K. Nagel ${ }^{b, c}$

a Integrated Program in Neuroscience, Douglas Mental Health Institute, McGill

University, Montreal, Quebec, H4H 1R3, Canada;

${ }^{\mathrm{b} H u m a n}$ Technology Center, RWTH Aachen University, 52062 Aachen, Germany

${ }^{\mathrm{C}}$ Department of Philosophy, University of Twente, 7500 AE Enschede

The Netherlands

daina.crafa@mail.mcgill.ca

saskia.nagel@humtec.rwth-aachen.de

http://www.dainacrafa.com

\begin{abstract}
Culture contextualizes the contents and intentionality of many mental statuses. Cognitive mediation of cultural information shapes these contents and intentionalities, as well as many of the false beliefs of pathology. Flexibility of cognitive mediation processes and resulting beliefs and pathologies may vary by individual, be a key mechanism of the feedback loop, and help characterize network connections.
\end{abstract}




\section{The adaptive self: Culture and social flexibility in feedback networks}

Many psychiatric disorders may be better explained by non-reductive, network models, as exemplified by Borsboom et al. in the target article, than by reductionist models that condense or dismiss distinct but interrelated cognitive processes. The influences of culture and individual social flexibility on mental disorders and outcomes are among the processes that network models can robustly account for. We agree with the authors' descriptions of the interplay between mental state contents and culture in symptom networks. To further enrich the discussion, we examine (1) the relevant details of the relationships between mental state contents and culture; (2) how these relationships might arise and feed back into themselves; and (3) the implications these relationships have for further defining appropriate network models.

Culture influences the causal relationship between symptoms in a network model. Culture also largely shapes the contents of mental states and intentionality of many mental states (e.g., Ambady \& Bharucha 2009; Ramos-Sánchez \& Atkinson 2009; Varela \& Shear 1999). The network relationships between symptoms appear causally related once their contents and intentionality are understood (Borsboom et al. in the target article, and see also Borsboom 2008). In the example by Borsboom et al. in the section on the Content of Mental States, someone who believes the Central Intelligence Agency (CIA) is watching will close the curtains. According to their line of argument, the CIA watching would be part of the cultural influence of being a patient in a country where the CIA is a relevant entity, such as the United States; however, the intentional act of closing the curtains and withdrawing from social life is also part of the cultural effects. These actions may be considered comprehensible in the United States, for example, because of the cultural interpretation of how to obtain safety from governmental agencies. Alternative actions, 
such as becoming hypersocial to find safety by remaining in a large group of people, may be equally understandable in a cultural context where groups are perceived as safer than isolation. This interpretation harmonizes recent findings in cultural psychiatry, such as variations in hallucination experiences of psychotic patients (Luhrmann et al. 2014), with findings in neuropsychiatry and on real-world patient behaviors (Bowie et al. 2008; Menon \& Uddin 2010).

Culture, of course, is characterized by numerous continua of common beliefs and behaviors (Kemmelmeier \& Kühnen 2012), and one must also ask how an individual decides which culturally informed behaviors to adhere to. Cognitive mediation of cultural information is arguably a key process that gives rise to the contents and intentionality of mental contents as well as many of the false beliefs that contribute to pathology (Kitayama \& Uskul 2011; Crafa \& Nagel, 2013, in press). Cognitive mediation in this context refers to the process of identity and belief construction based on consciously or unconsciously subscribing to or rejecting information in the sociocultural environment. Because social information is continuously encountered throughout the lifespan, this process is a feedback loop that constantly feeds into itself, but that also shapes the biological, neurological, and psychological constitution of the individual (Crafa \& Nagel, 2013, in press). New social experiences may reinforce or alter existing biological, neural, and psychological processes by providing both information and impetus. For example, the reification or undermining on the levels of existing beliefs, neural networks, or behavioral outputs changes what information in the social environment is experienced (e.g., other people respond differently to you depending on how you think and behave) and perceived (e.g., if you believe the CIA is after you, you will attend to different environmental information and interpret that information differently than you might otherwise) (Archpru Akaka \& Chandler 2011). The information in the social environment 
that is experienced and perceived then feeds back, either reinforcing or undermining existing processes, and the cycle continues ad mortem. This feedback loop shapes neural networks, behavioral outputs, and other biopsychological processes.

When examining the relationship between mental disorders and cognitively mediated feedback loops, individuals vary substantially in their abilities to adapt to novel or dynamically changing social situations (Folke et al. 2010). Social rigidity and hyperflexibility are symptoms of many disorders, such as obsessive-compulsive disorder, autism, and schizophrenia (Bliksted et al. 2014; Chamberlain et al. 2009; Geurts et al. 2009). From the perspective of a network model, it is useful to consider rigidity and hyperflexibility as parts of a single continuous trait of social flexibility. Where an individual falls on this continuum of social flexibility is informative for understanding whether exposure to new social information will reinforce or undermine existing processes to larger or smaller extents. Thus, in turn, an individual's degree of social flexibility may indicate how mental contents or intentionality might continue to develop across the lifespan and the magnitude of those changes. In other words, not all feedback loops are created equal. Considering the role of social flexibility as a key feature of any feedback loop can help further characterize the development, strength, and possible trajectories of network connections, and further specify how we can understand the complex reasoning of individual patients as well as the relationship between their reasoning and their underlying neurobiology.

The impact of culture on the contents of mental states and how a person cognitively mediates those experiences may vary depending on how flexible a person is. Understanding variations in human flexibility can be informative for characterizing and potentially predicting the 
impact network relations may have on the trajectory of individual mental states. Network models of psychiatric disorders will benefit from the inclusion of these interrelated processes in order to ultimately better understand the patient. 


\section{References}

Ambadyp, N. \& Bharucha, J. (2009) Culture and the brain. Current Directions in Psychological Science 18:342-45.

Archpru Akaka, M. \& Chandler, J. D. (2011) Roles as resources: A social roles perspective of change in value networks. Marketing Theory 11(3):243-60.

Bliksted, V., Fagerlund, B., Weed, E., Frith, C. \& Videbech, P. (2014) Social cognition and neurocognitive deficits in first-episode schizophrenia. Schizophrenia Research 153:9-17.

Borsboom, D. (2008) Psychometric perspectives on diagnostic systems. Journal of Clinical Psychology 9:1089-108.

Bowie, C. R., Leung, W. W., Reichenberg, A., McClure, M. M., Patterson, T. L., Heaton, R. K. \& Harvey, P. D. (2008) Predicting schizophrenia patients' real-world behavior with specific neuropsychological and functional capacity measures. Biological Psychiatry 63:505-11.

Chamberlain, S. R. \& Menzies, L. (2009) Endophenotypes of obsessive-compulsive disorder: Rationale, evidence and future potential. Expert Review of Neurotherapeutics 9:1133-46.

Crafa, D., \& Nagel, S. (2013). Accounting for Heterogeneity: The Culture-Brain-Behavior Interaction Model. Proceedings of the International Cultural Neuroscience Consortium (p. 4). Evanston, USA.

Crafa, D. \& Nagel, S. K. (in press) Traces of culture: The feedback loop between brain, behavior, and disorder. Transcultural Psychiatry.

Folke, C., Carpenter, S., Walker, B., Scheffer, M., Chapin, T. \& Rockström, J. (2010) Resilience thinking: Integrating resilience, adaptability and transformability. Ecology and Society 
15(4):article 20. Online publication. Available at:

http://www.ecologyandsociety.org/vol15/iss4/art20/

Geurts, H. M., Corbett, B. \& Solomon, M. (2009) The paradox of cognitive flexibility in autism. Trends in Cognitive Science 13:74-82.

Kemmelmeier, M. \& Kühnen, U. (2012) Culture as process: The dynamics of cultural stability and change. Social Psychology 43:171-73.

Kitayama, S. \& Uskul, A. K. (2011) Culture, mind, and the brain: Current evidence and future directions. Annual Review of Psychology 62:419-49.

Luhrmann, T.M., Padmavati, R., Tharoor, H. \& Osei, A. (2014) Differences in voice-hearing experiences of people with psychosis in the USA, India and Ghana: Interview-based study. British Journal of Psychiatry 206:41-44.

Menon, V. \& Uddin, L. Q. (2010) Saliency, switching, attention and control: A network model of insula function. Brain Structure and Function 214:655-67.

Ramos-Sánchez, L. \& Atkinson, D. R. (2009) The relationships between Mexican American acculturation, cultural values, gender, and help-seeking intentions. Journal of Counseling and Development 87:62-71.

Varela, F. J. \& Shear, J. (1999) First-person methodologies: What, why, how. Journal of Consciousness Studies 6:1-14. 